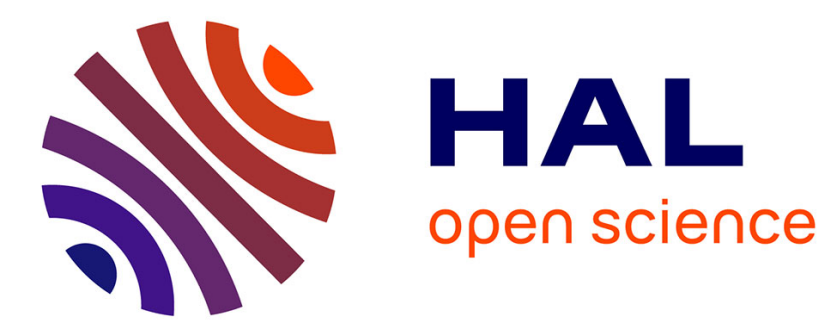

\title{
Transition to Turbulent Dynamo Saturation
}

Kannabiran Seshasayanan, Basile Gallet, Alexandros Alexakis

\section{To cite this version:}

Kannabiran Seshasayanan, Basile Gallet, Alexandros Alexakis. Transition to Turbulent Dynamo Saturation. Physical Review Letters, 2017, 119 (20), 10.1103/PhysRevLett.119.204503 . hal-01666655

\section{HAL Id: hal-01666655 https://hal.science/hal-01666655}

Submitted on 18 Dec 2017

HAL is a multi-disciplinary open access archive for the deposit and dissemination of scientific research documents, whether they are published or not. The documents may come from teaching and research institutions in France or abroad, or from public or private research centers.
L'archive ouverte pluridisciplinaire HAL, est destinée au dépôt et à la diffusion de documents scientifiques de niveau recherche, publiés ou non, émanant des établissements d'enseignement et de recherche français ou étrangers, des laboratoires publics ou privés. 


\title{
Transition to Turbulent Dynamo Saturation
}

\author{
Kannabiran Seshasayanan, ${ }^{1, *}$ Basile Gallet, ${ }^{2}$ and Alexandros Alexakis ${ }^{1}$ \\ ${ }^{1}$ Laboratoire de Physique Statistique, École Normale Supérieure, CNRS UMR 8550, Université Paris Diderot, \\ Université Pierre et Marie Curie, 24 rue Lhomond, 75005 Paris, France \\ ${ }^{2}$ Service de Physique de l'État Condensé, CEA, CNRS UMR 3680, Université Paris-Saclay, \\ CEA Saclay, 91191 Gif-sur-Yvette, France
}

(Received 4 April 2017; revised manuscript received 16 September 2017; published 17 November 2017)

While the saturated magnetic energy is independent of viscosity in dynamo experiments, it remains viscosity dependent in state-of-the-art 3D direct numerical simulations (DNS). Extrapolating such viscous scaling laws to realistic parameter values leads to an underestimation of the magnetic energy by several orders of magnitude. The origin of this discrepancy is that fully 3D DNS cannot reach low enough values of the magnetic Prandtl number Pm. To bypass this limitation and investigate dynamo saturation at very low Pm, we focus on the vicinity of the dynamo threshold in a rapidly rotating flow: the velocity field then depends on two spatial coordinates only, while the magnetic field consists of a single Fourier mode in the third direction. We perform numerical simulations of the resulting set of reduced equations for Pm down to $2 \times 10^{-5}$. This parameter regime is currently out of reach to fully 3D DNS. We show that the magnetic energy transitions from a high-Pm viscous scaling regime to a low-Pm turbulent scaling regime, the latter being independent of viscosity. The transition to the turbulent saturation regime occurs at a low value of the magnetic Prandtl number, $\mathrm{Pm} \simeq 10^{-3}$, which explains why it has been overlooked by numerical studies so far.

DOI: 10.1103/PhysRevLett.119.204503

The magnetic field of most astrophysical objects is believed to originate from the dynamo effect, an instability that converts part of the fluid kinetic energy into magnetic energy. The dynamo instability sets in when the flow is sufficiently vigorous to amplify magnetic field perturbations through electromagnetic induction and overcome Ohmic diffusion. In dimensionless form, this happens above a critical value $\mathrm{Rm}_{c}$ of the magnetic Reynolds number $\mathrm{Rm}=U \ell / \eta$, where $U$ and $\ell$ are the typical velocity and length scales of the flow and $\eta=1 / \mu_{0} \sigma$ is the magnetic diffusivity, with $\sigma$ the electrical conductivity of the fluid and $\mu_{0}$ the magnetic permeability of vacuum. An immediate difficulty arises from the low value of the magnetic Prandtl number $\operatorname{Pm}=\nu / \eta$, where $\nu$ is kinematic viscosity: values of Pm of the order of $10^{-5}$ are typical of liquid metals and solar system objects. As a consequence, when the flow reaches the $O(1)$ threshold value $\mathrm{Rm}_{c}$, the kinetic Reynolds number $\operatorname{Re}=U \ell / \nu$ is in the range $10^{5}-10^{6}$ and the flow is fully turbulent. This constitutes a challenge both experimentally and numerically: because of the high power needed to sustain a turbulent flow above $\mathrm{Rm}_{c}$, large experimental facilities are needed, and only three such experiments have succeeded in producing dynamo magnetic fields [1-3]. On the numerical side, direct numerical simulations (DNS) of the dynamo effect at realistic Pm values would require gigantic computational resources to accurately resolve the small scales of the fully turbulent flow. State-of-the-art dynamo DNS are therefore restricted to moderately low values of $\mathrm{Pm}$, typically $\mathrm{Pm} \geq 0.01$ in triple periodic boxes [4] and $\mathrm{Pm} \geq 0.05$ in spherical geometry [5-8].
Deriving and testing scaling laws that extrapolate numerical results to the physically motivated values of $\mathrm{Pm}$ is essential for relating DNS with observations [5,8-10]. In this quest for scaling relations, probing the dynamo effect at much lower Pm values is highly desirable, because the turbulence of the background flow strongly affects the magnetic energy produced above threshold. Consider, for instance, the vicinity of a supercritical dynamo bifurcation, a regime that is relevant to all dynamo laboratory experiments and possibly some planets: close to onset, one expects the magnetic energy to scale linearly with the departure from threshold $\left(\mathrm{Rm}-\mathrm{Rm}_{c}\right)$, with a dimensional prefactor that crucially depends on the value of the magnetic Prandtl number. Indeed, high-Pm dynamos and theoretical examples of laminar dynamo flows saturate through a balance between the Lorentz force and the viscous one. The magnetic energy above threshold then follows the "viscous" scaling law [11]:

$$
\frac{|\mathbf{B}|^{2} \ell^{2}}{\rho \mu_{0} \eta^{2}} \propto \operatorname{Pm}\left(\mathrm{Rm}-\mathrm{Rm}_{c}\right) .
$$

By contrast, laboratory experiments indicate that this saturation is achieved through a balance between the Lorentz force and the nonlinear advective term of the Navier-Stokes equation [12]. This leads to the "turbulent" scaling law for the magnetic energy [11]:

$$
\frac{|\mathbf{B}|^{2} \ell^{2}}{\rho \mu_{0} \eta^{2}} \propto\left(\mathrm{Rm}-\mathrm{Rm}_{c}\right),
$$


which corresponds to a much higher magnetic energy than the viscous scaling law (1) by a factor $\mathrm{Pm}^{-1}$.

An interesting approach to test these theoretical predictions is the one based on shell models of MHD turbulence. Such phenomenological models are not meant to quantitatively describe the flows, but rather to capture their statistical properties at reasonable computational cost [13]: they provide evidence for the scaling-law (2) when the magnetic Prandtl number is low enough, Pm $\lesssim 1$ [14].

The natural next step towards quantitative numerical dynamo models would be to reproduce the turbulent scaling regime directly from numerical solutions of the Navier-Stokes and induction equations, a task which remains beyond reach of state-of-the-art fully 3D DNS. Indeed, it has been recently shown that all spherical dynamo simulations obey the viscous scaling law $[8,10]$ : because of the moderately low Pm, they are restricted to the viscosity-dominated regime, which makes their extrapolation to Earth-like parameters questionable. A central question is, therefore, how much smaller need the magnetic Prandtl number be to start observing the turbulent scaling regime (2)?

We address this question by focusing on rapidly rotating flows in the vicinity of the dynamo threshold. Global rotation is a relevant ingredient of many planetary dynamos, which strongly affects the dynamo characteristics [15]. For rapid global rotation, we are able to reduce the full MHD system to a set of quasi-2D equations governing the interaction between a two-dimensional three-component (2D3C) flow and a vertically dependent dynamo magnetic field. This approach allows us to bypass the current limitations of 3D DNS: we focus on the high-Reynoldsnumber regime, where nonlinear advection strongly dominates over viscous effects, in a quasi-2D system of manageable computational cost.

Reduced equations. - We consider a flow driven by a vertically invariant body force $\mathbf{f}(x, y)$ in a frame rotating at a rate $\Omega$ around the vertical $z$ axis. It was recently proven that the corresponding 2D turbulent flow is stable to 3D perturbations provided the Rossby number is sufficiently low [16]. We focus on this parameter range, the turbulent 2D3C velocity field $\mathbf{u}=(u(x, y, t), v(x, y, t), w(x, y, t))$ being the base flow of the present dynamo study. For the kinematic dynamo problem, the invariance of the flow along the vertical direction allows us to decompose the magnetic field into vertical Fourier modes and to study their evolution independently:

$$
\mathbf{B}(x, y, z, t)=\mathbf{b}(x, y, t) e^{i k z}+\text { c.c. },
$$

where c.c. denotes the complex conjugate and $k$ the vertical wave number. Inserting such a Fourier mode into the induction equation leads to the evolution equation for $\mathbf{b}$ :

$$
\partial_{t} \mathbf{b}=\left(\boldsymbol{\nabla}_{\perp}+i k \mathbf{e}_{z}\right) \times(\mathbf{u} \times \mathbf{b})+\eta\left(\boldsymbol{\nabla}_{\perp}^{2}-k^{2}\right) \mathbf{b},
$$

where $\boldsymbol{\nabla}_{\perp}=\left(\partial_{x}, \partial_{y}, 0\right)$ and $\mathbf{e}_{z}$ is the unit vector along $z$ [17-19].

We focus on the weakly nonlinear regime in the vicinity of the dynamo threshold, for which we can keep only the first unstable magnetic mode of the form (3). The instability saturates through the action of the Lorentz force. The latter being quadratic in the magnetic field (3), it contains two harmonics in $z$ : (i) A $z$-independent component (harmonic 0), through which the magnetic field directly feeds back on to the $z$-invariant base flow. (ii) A second harmonic component, with vertical dependence $e^{ \pm 2 i k z}$. It forces a second harmonic in the velocity field, $\mathbf{u}_{2}=$ $\hat{\mathbf{u}}_{2}(x, y, t) e^{2 i k z}+$ c.c., the amplitude of which results from a balance between the Coriolis and Lorentz forces:

$$
\mathbf{u}_{2} \sim \frac{\mathbf{B}^{2}}{\rho \mu_{0} \ell \Omega} .
$$

The vertical average of the Coriolis force being absorbed by the pressure gradient, the $z$-independent flow follows the equation

$$
\begin{aligned}
\partial_{t} \mathbf{u}+\left(\mathbf{u} \cdot \boldsymbol{\nabla}_{\perp}\right) \mathbf{u}= & -\boldsymbol{\nabla}_{\perp} p-\gamma \mathbf{u}_{\perp}+\nu \nabla_{\perp}^{2} \mathbf{u}+\mathbf{f}(x, y) \\
& +\frac{1}{\rho \mu_{0}}\left\{\left[\left(\nabla_{\perp}+i k \mathbf{e}_{z}\right) \times \mathbf{b}\right] \times \mathbf{b}^{*}+\text { c.c. }\right\},
\end{aligned}
$$

where $\mathbf{u}_{\perp}=(u, v, 0), \gamma$ is a linear Ekman friction coefficient [20], and the last term is the vertical average of the Lorentz force. The latter induces a correction to the turbulent $2 \mathrm{D} 3 \mathrm{C}$ base flow of order $\mathbf{B}^{2} /\left(\rho \mu_{0} U\right)$. By contrast, Eq. (5) indicates that $\mathbf{u}_{2}$ is smaller than this correction by a factor equal to the Rossby number $U / \ell \Omega$, which is asymptotically small in the present study: we therefore discard $\mathbf{u}_{2}$ in the following. The weakly nonlinear regime in the vicinity of the dynamo threshold then corresponds to the interaction between the first unstable vertical Fourier mode of the magnetic field and the $z$-invariant 2D3C flow. Their coupled evolution obeys the reduced system of equations (4)-(6).

Numerical experiments. - We solve Eqs. (4)-(6) inside a domain $(x, y) \in[0,2 \pi L]^{2}$ using standard pseudospectral methods $[18,21]$. The body force has the Roberts-flow geometry,

$\mathbf{f}(x, y)=F(\cos (y / \ell), \sin (x / \ell), \cos (x / \ell)+\sin (y / \ell))$,

where the scale of the forcing is $\ell=L / 4$. This value was shown to be close to optimal for reducing the dynamo threshold [22], which is desirable to reach the lowmagnetic-Prandtl-number regime.

The vertical wave number is set to $k=0.2 / L$, which corresponds to the lowest wave number inside a domain of height $10 \pi L$. The influence of this parameter on the dynamo threshold and magnetic field geometry was studied 


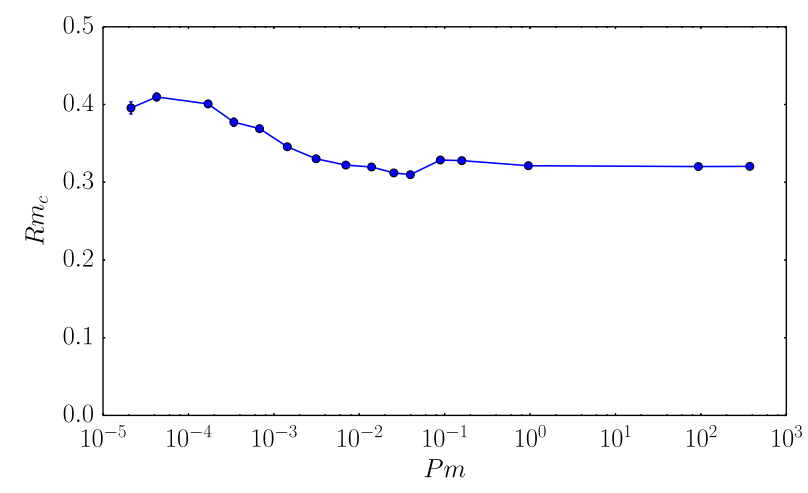

FIG. 1. Threshold magnetic Reynolds number $\mathrm{Rm}_{c}$ for dynamo action as a function of $\mathrm{Pm}$, for $\gamma \ell^{2} / \eta=5.1 \times 10^{-3}$.

in detail in a previous publication [18], the phenomenology being that of standard $\alpha^{2}$ dynamos $[23,24]$.

We first solve the purely hydrodynamic problem until a statistically steady state is reached. We denote as $U$ the root-mean-square velocity of this state that depends on $F, \nu$, $\gamma$. This flow is then used as the starting point of the MHD simulations. We solve the MHD problem for increasing values of $F$, i.e., for increasing values of the magnetic Reynolds number Rm while keeping $\operatorname{Pm}$ fixed $(\nu, \gamma$, and $\eta$ are fixed). Above the threshold value $\mathrm{Rm}_{c}$ for dynamo action, the dynamo instability sets in and magnetic perturbations grow. As shown in Fig. $1, \mathrm{Rm}_{c}$ varies little with $\mathrm{Pm}$, with $\mathrm{Rm}_{c} \in$ [0.30; 0.42] over seven decades of Pm. Such a constant value of $\mathrm{Rm}_{c}$ at low $\mathrm{Pm}$ is rather generic and has been reported for several other forcing geometries $[25,26]$. By contrast, the behavior of $\mathrm{Rm}_{c}$ in the transitional region of intermediate $\mathrm{Pm}$ strongly depends on the structure of the forcing: for some fully 3D flows with weak scale separation, $\mathrm{Rm}_{c}$ displays a twofold increase at intermediate $\mathrm{Pm}$ [24]. The weak variation of $\mathrm{Rm}_{c}$ with $\mathrm{Pm}$ in Fig. 1 is, therefore, attributed to both the scale separation and the 2D3C nature of the flow $[22,27]$.

After the initial growth phase, the magnetic field saturates through the feedback of the Lorentz force onto the 2D3C flow. In Fig. 2, we show snapshots of the velocity and magnetic fields in the saturated state, for $\mathrm{Pm}=4.25 \times 10^{-5}$. In agreement with standard $\alpha^{2}$-dynamo theory [23], the vertical velocity and vorticity are concentrated at the forcing scale $\ell$ while the dynamo magnetic field is at large scale, with horizontal components more energetic than the vertical one. We compute the space and time averaged magnetic energy in the saturated state, to produce bifurcation curves such as the ones shown in Fig. 3. We repeat this procedure for various values of the magnetic Prandtl number Pm, and from each bifurcation curve we extract the slope $S(\mathrm{Pm})$ relating the magnetic energy to the departure from onset:

$$
\frac{\left\langle|\mathbf{B}|^{2}\right\rangle \ell^{2}}{\rho \mu_{0} \eta^{2}}=S(\mathrm{Pm}) \times\left(\mathrm{Rm}-\mathrm{Rm}_{c}\right),
$$
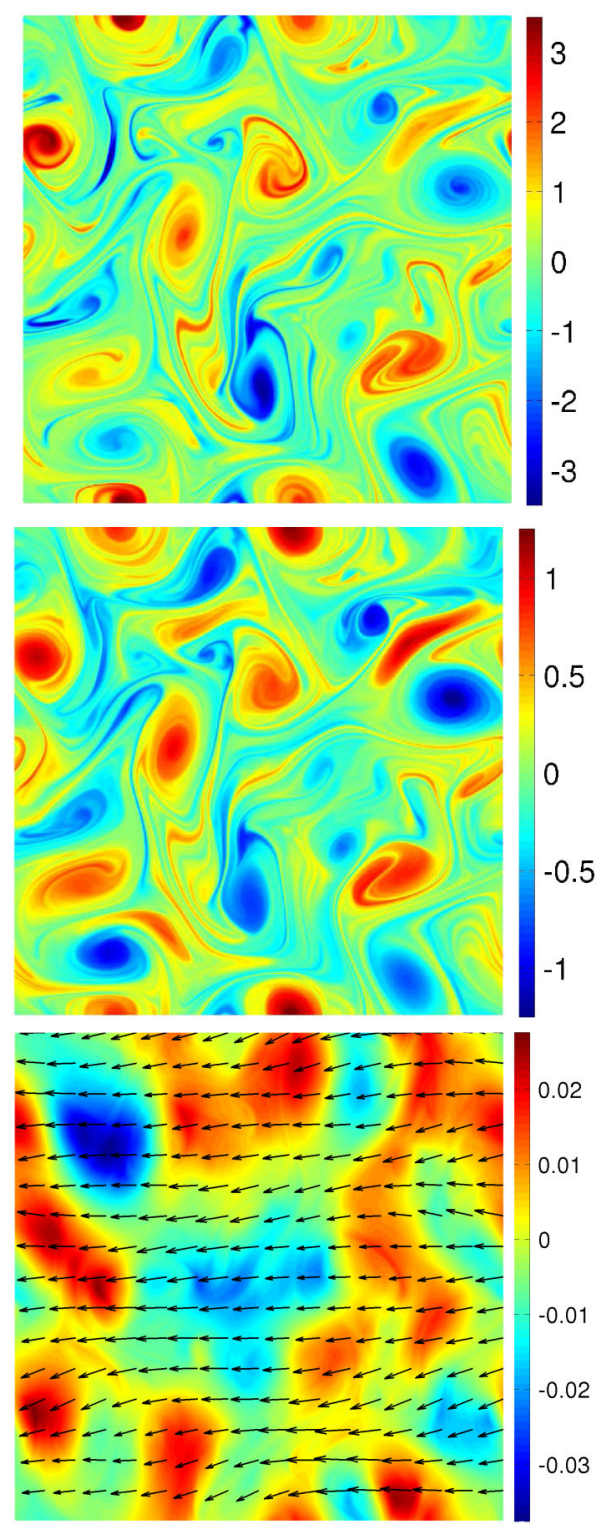

FIG. 2. Snapshots of the saturated state for $\mathrm{Pm}=4.25 \times 10^{-5}$, $\gamma \ell^{2} / \eta=5.1 \times 10^{-3}$, and $\mathrm{Rm}=0.44$. Top: vertical vorticity in units of $U / \ell$. Middle: vertical velocity in units of $U$. Bottom: dimensionless magnetic field $\mathbf{B} \ell / \sqrt{\rho \mu_{0} \eta^{2}}$ in the plane $z=0$. The arrows indicate the horizontal components while color codes for the vertical one. These arrows correspond to a typical magnitude $4 \times 10^{-2}$ of the dimensionless horizontal magnetic field. Their average direction rotates with $z$.

where the angular brackets denote $3 \mathrm{D}$ space and time average. $S(\mathrm{Pm})$ is the central quantity of the present study.

From viscous to turbulent saturation.--In Fig. 4, we show $S(\mathrm{Pm})$ over seven decades of Pm. For large Pm, the flow is laminar and has a low Reynolds number near the dynamo threshold. Accordingly, the dynamo saturation obeys the viscous scaling-law (1), i.e., $S(\mathrm{Pm}) \sim \mathrm{Pm}$ for large $\mathrm{Pm}$. The precise expression of $S(\mathrm{Pm})$ can be determined analytically in the limit of scale separation $k \ell \ll 1$ and corresponds to the usual viscous alpha 


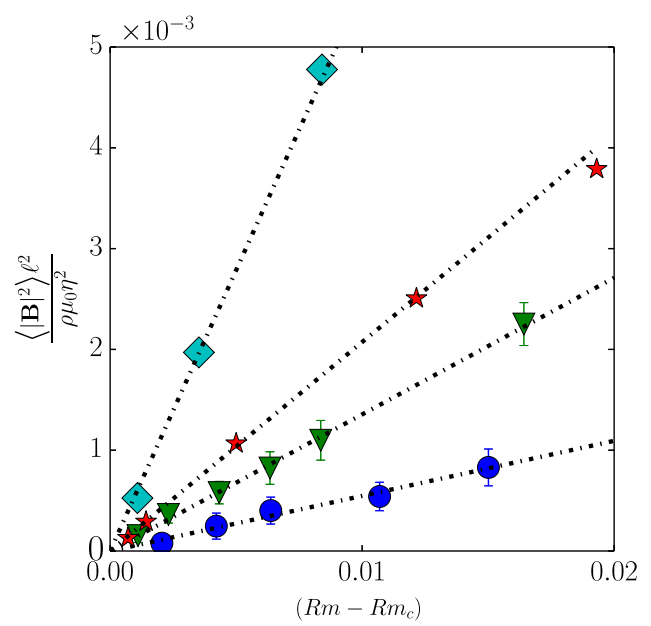

FIG. 3. Magnetic energy as a function of the departure from onset, for several values of the magnetic Prandtl number. The friction is $\gamma \ell^{2} / \eta=5.1 \times 10^{-3}$ and symbols are circle, $\mathrm{Pm}=$ $1.4 \times 10^{-3}$; inverted triangle, $\mathrm{Pm}=7.0 \times 10^{-3}$; star, $\mathrm{Pm}=$ $4.0 \times 10^{-2}$; open diamond, $\mathrm{Pm}=8.9 \times 10^{-2}$. The dashed linear fits allow to extract the slope $S(\mathrm{Pm})$ of each bifurcation curve.

quenching. For small friction $\gamma \ell^{2} \ll \nu$, following the standard weakly nonlinear approach $[28,29]$ we obtain

$$
S(\mathrm{Pm})=\sqrt{\frac{2}{k \ell}} \mathrm{Pm} .
$$

This analytical prediction is displayed in Fig. 4 with a solid line and captures perfectly the high-Pm behavior of $S(\mathrm{Pm})$.

In contrast with such viscous dynamos, $S(\mathrm{Pm})$ reaches a plateau at low Pm, with values orders of magnitude larger than predicted by the laminar theory. This corresponds to the turbulent scaling-law (2), for which $S(\mathrm{Pm})$ is independent of Pm. More precisely, in this regime $S$ is independent of both $\nu$ and $\gamma$ (see inset of Fig. 4) and the dominant balance in the Navier-Stokes equation (6) is between the Lorentz force and the nonlinear term. To our knowledge, this study constitutes the first numerical observation of the turbulent scaling regime of dynamo saturation in a solution of the coupled Navier-Stokes and induction equations. This is because extremely low values of $\mathrm{Pm}$ are needed to observe such turbulent saturation: the plateau in figure 4 arises for $\mathrm{Pm} \lesssim 10^{-3}$, an order of magnitude below the smallest values of Pm achieved in state-of-the-art fully 3D DNS.

Discussion.-We have reported the numerical observation of the turbulent scaling regime for dynamo saturation in a solution of the MHD equations. Our work, therefore, quantifies for the first time previous theoretical estimates based on dimensional analysis [11] and shell models [14]: in the present setup, the turbulent scaling regime sets in for values of the magnetic Prandtl number an order of magnitude lower than currently achieved by

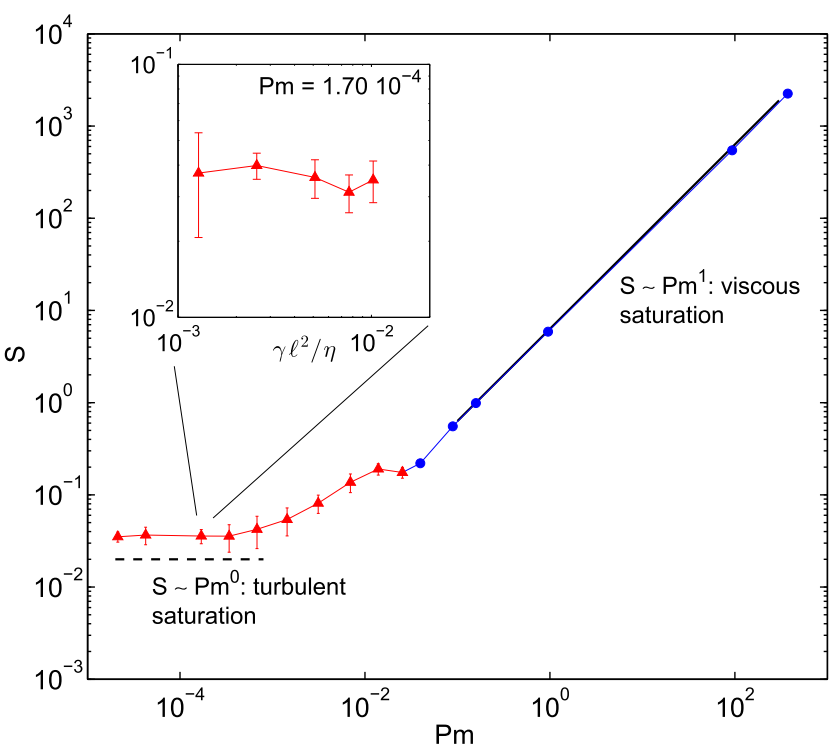

FIG. 4. Slope $S$ of the magnetic energy above onset. High-Pm solutions are time independent (blue circles) and obey the quantitative prediction (9) from viscous alpha quenching (thick solid line). For low Pm, the flow is time dependent (red triangles). For $\mathrm{Pm} \lesssim 10^{-3}$, the dynamo saturation follows the turbulent scaling-law (2), represented as a dashed eye guide. The main figure corresponds to a friction coefficient $\gamma \ell^{2} / \eta=5.1 \times 10^{-3}$. The inset highlights the independence of $S$ on friction in the turbulent saturation regime.

state-of-the-art fully 3D DNS. This explains the mismatch between spherical dynamo simulations, which obey the viscous scaling law [10], and experimental dynamos, which follow the turbulent one [12]. Because the turbulent scaling regime is likely to be the generic situation for many natural and experimental dynamos, this study paves the way for quantitative numerical estimates of the magnetic field in astrophysical objects and laboratory experiments.

This work was granted access to the HPC resources of GENCI-CINES (Projects No. x2014056421, No. x2015056421) and MesoPSL financed by the Region Ile de France and the project EquipMeso ANR-10-EQPX29-01. K. S. acknowledges support from LabEx ENS-ICFP: ANR-10-LABX-0010/ANR-10-IDEX-0001-02 PSL and from École Doctorale Île de France (EDPIF). B. G. acknowledges support from LabEx PALM ANR-10-LABX-0039.

*skannabiran@1ps.ens.fr

[1] R. Stieglitz and U. Müller, Phys. Fluids 13, 561 (2001).

[2] A. Gailitis, O. Lielausis, E. Platacis, S. Dement'ev, A. Cifersons, G. Gerbeth, T. Gundrum, F. Stefani, M. Christen, and G. Will, Phys. Rev. Lett. 86, 3024 (2001).

[3] R. Monchaux, M. Berhanu, M. Bourgoin, M. Moulin, P. Odier, J.-F. Pinton, R. Volk, S. Fauve, N. Mordant, F. Pétrélis et al., Phys. Rev. Lett. 98, 044502 (2007).

[4] P. D. Mininni, Phys. Rev. E 76, 026316 (2007). 
[5] U. R. Christensen and J. Aubert, Geophys. J. Intl. 166, 97 (2006).

[6] U. Christensen, Space Sci. Rev. 152, 565 (2010).

[7] C. A. Jones, Annu. Rev. Fluid Mech. 43, 583 (2011).

[8] E. King and B. Buffett, Earth Planetary Sci. Lett. 371, 156 (2013).

[9] P. Davidson, Geophys. J. Int. 195, 67 (2013).

[10] L. Oruba and E. Dormy, Geophys. J. Int. 198, 828 (2014).

[11] F. Pétrélis and S. Fauve, Eur. Phys. J. B 22, 273 (2001).

[12] F. Pétrélis, N. Mordant, and S. Fauve, Geophys. Astrophys. Fluid Dyn. 101, 289 (2007).

[13] F. Plunian, R. Stepanov, and P. Frick, Phys. Rep. 523, 1 (2013).

[14] R. Stepanov and F. Plunian, J. Turbul. 7, N39 (2006).

[15] J. Donati, Symp. - Int. Astron. Union 215, 258 (2004).

[16] B. Gallet, J. Fluid Mech. 783, 412 (2015).

[17] S. G. L. Smith and S. Tobias, J. Fluid Mech. 498, 1 (2004).

[18] K. Seshasayanan and A. Alexakis, J. Fluid Mech. 799, 246 (2016).

[19] K. Seshasayanan and A. Alexakis, J. Fluid Mech. 806, 627 (2016).
[20] J. Pedlosky, Geophysical Fluid Dynamics (Springer, New York, 1979).

[21] D. O. Gomez, P. D. Mininni, and P. Dmitruk, Phys. Scr. 116, 123 (2005).

[22] M. Sadek, A. Alexakis, and S. Fauve, Phys. Rev. Lett. 116, 074501 (2016).

[23] H. Moffatt, Magnetic Field Generation in Electrically Conducting Fluids (Cambridge University Press, Cambridge, England, 1978).

[24] Y. Ponty and F. Plunian, Phys. Rev. Lett. 106, 154502 (2011).

[25] Y. Ponty, P. Mininni, D. Montgomery, J.-F. Pinton, H. Politano, and A. Pouquet, Phys. Rev. Lett. 94, 164502 (2005).

[26] A. Iskakov, A. Schekochihin, S. Cowley, J. McWilliams, and P. M. R. E., Phys. Rev. Lett. 98, 208501 (2007).

[27] K. Seshasayanan, V. Dallas, and A. Alexakis, arXiv: 1701.08996.

[28] A. D. Gilbert and P.-L. Sulem, Geophys. Astrophys. Fluid Dyn. 51, 243 (1990).

[29] S. Fauve and F. Pétrélis, Peyresq Lectures on Nonlinear Phenomena (World Scientific, Singapore, 2003). 